\title{
Cerebroprotective effect of xantohumol sensu experimental stroke model: study design and preliminary results
}

\author{
Aigul R. Saitgareyeva ${ }^{1}$, Leyla R. Akhmadeyeva ${ }^{1}$ \\ ${ }^{1}$ Bashkir State Medical University
}

Received 5 February 2020, Accepted 25 February 2021

Original Text (C) Saitgareyeva A.R., Akhmadeyeva L.R., 2020, published in Saratov Journal of Medical Scientific Research 2020; 16 (1): 377379 .

(C) 2021, Saitgareyeva A.R., Akhmadeyeva L.R.

(C) 2021, Saratov Medical Journal

\begin{abstract}
:
The objective of our study was to evaluate the cerebroprotective effect of xanthohumol (XN) on experimental models of acute ischemic stroke in vivo and in vitro.

Materials and methods. We used middle cerebral artery occlusion (MCAO) and oxygen-glucose deprivation (OGD) as in vivo and in vitro models. Our study subjects were Sprague-Dawley rats, which were randomly assigned to three groups: the control group and two MCAO groups with and without XN. The primary culture of cortical neurons was obtained from newborn rats. We employed the Bederson test and the corner test to evaluate neurological disorders.

Results. The preliminary results indicated a possible cerebroprotective effect of XN in an ischemic stroke model.

Conclusion. Preventive administration of $\mathrm{XN}$ before cerebral ischemia in an experiment can effectively reduce the volume of cerebral infarction and improve neurologic deficit 24 hours after MCAO.
\end{abstract}

Keywords: ischemic stroke, xanthohumol, experimental model

Cite as Saitgareyeva AR, Akhmadeyeva LR. Cerebroprotective effect of xantohumol sensu experimental stroke model: study design and preliminary results. Saratov Medical Journal 2021; 2(1): e0103.

Correspondence to Aigul R. Saitgareyeva, Tel.: +7(987)2504888. E-mail: aneuro@yandex.ru

\section{Introduction}

Stroke remains a global health problem, especially in undeveloped countries [1]. Clinically, it is the second leading cause of death [2], making its prevention and treatment a worldwide public healthcare issue. Ischemic stroke accounts for the majority of cases, while the rest are represented by hemorrhagic stroke. Ischemic stroke is also the main cause of disability in the world [3], as a result of which, the quality of life in both the patient and his/her live-in relatives and friends degrades. This fact makes ischemic stroke an urgent problem of both medical and socioeconomic importance.

The ischemic cascade is controlled by molecular mechanisms that trigger the destruction of the blood-brain barrier (BBB), contributing to the secondary progression of injury by means of exacerbation of cerebral edema and inflammatory response in its subacute phase [4]. The severity of these early events reduces the ability of neurons to recover in the chronic phase, significantly worsening stroke outcomes [5]. It is imperative to point out that, as a result of ischemic stroke, two main areas of damage arise: the nucleus, or infarction, undergoing immediate cell death as a result of necrosis; and peri-infarct penumbra, involving delayed programmed cell death [6]. The slow progression of cell death within the penumbra suggests that therapeutic rescue is possible. However, despite significant progress in stroke prevention and supportive care, there is no disease-modifying therapy as yet. Until now, induced thrombolysis with the use of recombinant tissue plasminogen activator (rt-PA) remains the only Food and Drug Administration (FDA) approved approach, used in Russian clinics and around the world to restore the cerebral blood flow. The main challenge in stroke therapy is finding an effective cerebroprotective agent capable of rescuing neurons in a potentially salvable transition zone (penumbra) that expands after the brain damage. Therefore, finding alternative stroke therapy with good efficacy and safety remains a high priority.

Xanthohumol (2',4',6',4-tetrahydroxy-3'-prenylchalcone) is the main prenylated chalcone (open C-ring flavonoid) from common hops (Humulus lupulus L.). The structure of xanthohumol (XN) was first revealed by M. Verzel with colleagues in 1957 [7]. However, beneficial pharmacological properties of $\mathrm{XN}$, including its antioxidant, antiinflammatory, antibacterial, antiviral and antifungal activity, were not fully elucidated until the 1990s. [8]. In traditional Chinese medicine, hops are used to treat insomnia, anxiety, indigestion, and lack of appetite. Alcohol extracts of hops have been successfully used in China for treating leprosy, pulmonary tuberculosis, acute bacterial dysentery, as well as in anticancer therapy [9]. Recently, XN has attracted considerable interest due to its biological activity, including possible cerebroprotective properties [10].

Objective: To evaluate the cerebroprotective effect of xanthohumol on experimental models of acute ischemic stroke in vivo and in vitro. 


\section{Materials and methods}

The in vivo study included 36 male Sprague-Dawley rats with body weights of 250-280 g. All experiments were conducted in compliance with the methodological guidelines for the care and use of laboratory animals at the national institutions of health. The animals were randomly assigned to three groups: the control group $(n=12)$, which underwent surgery according to the middle cerebral artery occlusion (MCAO) protocol, but without vessel occlusion; and two treatment groups encompassing the animals operated on with MCAO $(n=24)$, one of which $(n=12)$ was injected $0.4 \mathrm{mg}$ of XN per $1 \mathrm{~kg}$ of its body weight intraperitoneally 10 minutes before MCAO. Rats in the control group and the MCAO group received saline injection as a placebo. For anesthesia, the animals were injected with chloral hydrate $(350 \mathrm{mg} / \mathrm{kg}$ body weight) intraperitoneally. Both MCAO groups were subjected to MCAO surgery [11]. After 60 minutes, the monofilament was gently removed, and the rats were reperfused for 24 hours. Rats in the control group were also operated within the experimental MCAO model, but without the introduction of a monofilament. Animals were examined for neurologic deficits using the Bederson scale and the corner test [12]. These tests were conducted before MCAO and 24 hours after it. For the corner test, a corner with a 30-degree angle was prepared, and then the rats were allowed to enter that corner. The direction of rats turned at an angle was registered. Ischemic animals were characterized by developing the neglect of the space on their sick side; hence they were turning mainly in the healthy direction. The number of turns in each direction per 10 attempts was counted. The Bederson test was carried out three times, and we took into account the neurologic deficit of over 2 points on this test. $P$ values less than 0.05 were considered significant.

The primary culture of neurons for the in vitro component of our study was obtained from newborn rats. The cells were suspended in nerve-based medium containing 2\% B27 and $10 \%$ fetal bovine serum, and were placed on a culture dish coated with polylysine; then they were placed in an incubator containing $5 \%$ carbon dioxide at $37{ }^{\circ} \mathrm{C}$. The medium was changed every three days [13]. Cultures of neurons were randomly divided into three groups: the control group, and two oxygen-glucose deprivation (OGD) groups - with and without XN.

To measure the infarction volume for the in vivo model, the following steps were taken: after 24-hour reperfusion, the rats were sacrificed, then their brain was perfused with cold PBS (phosphate buffered saline, $\mathrm{pH}=7.4$ ), and then perfused with cold 4\% polyformaldehyde (PFA). Brain tissue was immersed in sucrose and cut into frozen coronary sections 20 $\mu \mathrm{m}$ thick. To determine the volume of cerebral infarction, brain cross-sections were stained with hematoxylin and eosin and observed under an optical microscope. To monitor the number and morphology of neurons, Nissl staining was performed. Frozen brain cross-sections were stained sensu Nissl, and then decolorized with alcohol and immersed in xylene in compliance with the manufacturer instructions. The observation was carried out using optical microscopy.

\section{Results}

The XN-treated group of rats exhibited significantly better results on the Bederson test and corner test 24 hours after the stroke, when compared with MCAO group without $\mathrm{XN}$. The difference in neurologic deficit values between the groups was 1 point on Bederson scale. The use of corner test in ischemic rats in MCAO and XN groups revealed that the animals developed motor neglect in the right half of the space; therefore, in the corner test, such rats were turning mainly to the left. In addition, it was discovered that rats, receiving $\mathrm{XN}$, had a slightly higher survival rate than rats in the untreated group: the survival rate in the $\mathrm{XN}$ group was 92\%, vs. $70 \%$ in the MCAO group. Under post-mortem staining of cerebral structures with hematoxylin and eosin to determine the infarction volume, the most massive cerebral infarction was observed in the MCAO group that did not receive $\mathrm{XN}$ injection (Figure).

The volume of cerebral infarction was significantly reduced when $\mathrm{XN}$ was administered to rats prior to MCAO surgery. These data indicate that pretreatment with $\mathrm{XN}$ provides the cerebroprotective effect.

Nissl staining was performed to monitor morphological changes of ischemic neurons. The neurons in the control group did not undergo ischemia. Neurons were becoming dark and triangular in the MCAO group without $\mathrm{XN}$, suggesting neuronal damage. The morphology of neurons in the $\mathrm{XN}$ group was to a greater extent similar to the shape of neurons observed in the control group. Flow cytometry was then performed to assess the effect of $\mathrm{XN}$ on neuronal viability. Analysis of cell viability using CCK-8 showed that neuronal viability was significantly reduced after OGD. The introduction of $\mathrm{XN}$ increased the viability and survival of neurons after OGD. Besides, the rate of neuronal apoptosis induced by OGD was significantly higher compared with the control group, while the rate of neuronal apoptosis in the $\mathrm{XN}$ group was significantly lower.

\section{Discussion}

Animal models of focal cerebral ischemia, for which MCAO is habitually used, reproduce the pattern of ischemic brain damage observed in many human patients with ischemic stroke [14]. In ischemic brain cells, neuronal cell death is irreversible [15]. The possible efficacy of various interventions before the onset of cerebral infarction is considered, which may provide prospective patients with the advantage of a favorable outcome in cerebral ischemia. XN has many pharmacological effects and is considered a potential natural beneficial agent. Although $\mathrm{XN}$ has been shown to have cerebroprotective potential, its beneficial effects on cerebral ischemia and stroke remain largely unclear [16] and require more detailed studying. It is also planned to conduct western blotting to identify the quantitative level of protein expression in brain tissue homogenates and primary neurons.

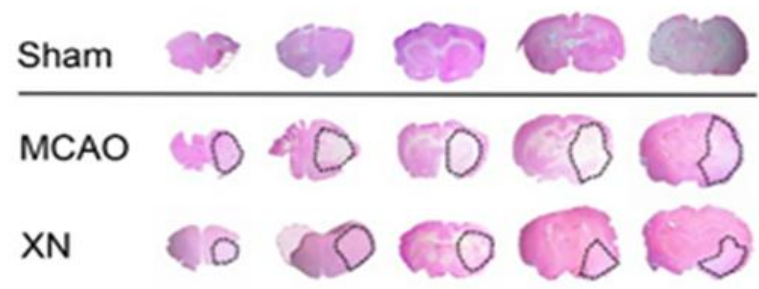

Figure. The volume of cerebral infarction in the experiment. Hematoxylin and eosin staining

Sham - control group; MCAO - group of rats with middle cerebral artery occlusion;

XN - group of rats with middle cerebral artery occlusion and use of xanthohumol 


\section{Conclusion}

At the stage of preliminary results, we established that preventive administration of XN prior to cerebral ischemia in the experiment can effectively reduce the volume of cerebral infarction and improve neurologic deficit 24 hours after MCAO. In addition, the use of XN increases the viability of animals in the experiment and survival of neurons after OGD. In the future, we intend to proceed with this study in order to collect more data to confirm the XN cerebroprotective effect.

\section{Acknowledgements}

The study was funded by Bashkir State Medical University (Russia) and Harbin Medical University (China).

\section{Conflict of interest}

The authors declare no conflict of interest.

\section{References}

1. O'Donnell MJ, Chin SL, Rangarajan S, et al. Global and regional effects of potentially modifiable risk factors associated with acute stroke in 32 countries (INTERSTROKE): A case-control study. Lancet 2016; (388): 761-75. https://doi.org/10.1016/s01406736(16)30506-2

2. Lozano R, Naghavi M, Foreman K, et al. Global and regional mortality from 235 causes of death for 20 age groups in 1990 and 2010: A systematic analysis for the Global Burden of Disease Study 2010. Lancet 2012; (380): 2095-128. https://doi.org/10.1016/So140-6736(12)61728-o

3. Mendis S, Davis S, Norrving B. Organizational update: the world health organization global status report on noncommunicable diseases 2014; one more landmark step in the combat against stroke and vascular disease. Stroke 2015; (46): e121-2. https://doi.org/10.1161/STROKEAHA.115.008097

4. Geissmann F, Manz MG, Jung S, et al. Development of monocytes, macrophages, and dendritic cells. Science 2010; (327): 656-61. https://doi.org/10.1126/science.1178331

5. Elali A. The implication of neurovascular unit signaling in controlling the subtle balance between injury and repair following ischemic stroke. Neural Regen Res 2016; (11): 914-5. https://doi.org/10.4103/1673-5374.184485

6. Lo EH. A new penumbra: Transitioning from injury into repair after stroke. Nat Med 2008; (14): 497-500. https://doi.org/10.1038/nm1735

7. Verzele M, Stockx J, Fontijn F, Anteunis M. Xanthohumol, a new natural chalkone. $J$ Agric Food Chem 1957; (66): 452-75.

8. Liu M, Hansen PE, Wang G, et al. Pharmacological profile of xanthohumol, a prenylated flavonoid from hops (Humulus $\begin{array}{llll}\text { lupulus). } \quad \text { Molecules 2015; (20): } & \text { 754-79. }\end{array}$ https://doi.org/10.3390/molecules20010754

9. Zanoli P, Zavatti M. Pharmacognostic and pharmacological profile of Humulus lupulus L. J Ethnopharmacol 2008; (116): 383-96. https://doi.org/10.3390/molecules20010754

10. Xuan NT, Shumilina E, Gulbins EGu, et al. Triggering of dendritic cell apoptosis by xanthohumol. Mol Nutr Food Res 2010; $54 \quad$ (Suppl. 2 2): https://doi.org/10.1002/mnfr.200900324

11. Chen B, Zhang F, Li QY, Gong A, Lan Q. Protective effect of AdVEGF-Bone mesenchymal stem cells on cerebral infarction. Turk Neurosurg 2016; (26): 8-15. https://doi.org/10.5137/10195149.JTN.11488-14.3

12. Bederson JB, Pitts LH, Tsuji M, et al. Rat middle cerebral artery occlusion: Evaluation of the model and development of a neurologic examination. Stroke 1986; (17): 472-6. https://doi.org/10.1007/BFo1403007
13. Qu Y, Mao M, Zhao F, et al. Proapoptotic role of human growth and transformation-dependent protein in the developing rat brain after hypoxia-ischemia. Stroke 2009; (40): 2843-8. https://doi.org/10.1161/STROKEAHA.109.553644

14. Ginsberg MD, Busto R. Rodent models of cerebral ischemia. Stroke 1989, $\quad$ (20): $1627-42$. https://doi.org/10.1161/01.str.20.12.1627

15. Iadecola C, Anrather J. The immunology of stroke: From mechanisms to translation. Nat Med 2011; (17): 796-808. https://doi.org/10.1038/nm.2399

16. Li F, Yao Y, Huang H, et al. Xanthohumol attenuates cisplatininduced nephrotoxicity through inhibiting NF- $\mathrm{kB}$ and activating Nrf2 signaling pathways. Int Immunopharmacol 2018; (61): 27782. https://doi.org/10.1016/j.intimp.2018.05.017

\section{Authors:}

Aigul R. Saitgareyeva - PhD student, Department of Neurology, Bashkir State Medical University, Bashkiria, Russia;

Leyla R. Akhmadeyeva - DSc, Professor, Department of Neurology, Bashkir State Medical University, Bashkiria, Russia. 